Tropical Journal of Pharmaceutical Research August 2020; 19 (8): 1653-1659

ISSN: $1596-5996$ (print); 1596-9827 (electronic)

(C) Pharmacotherapy Group, Faculty of Pharmacy, University of Benin, Benin City, 300001 Nigeria.

Original Research Article

http://dx.doi.org/10.4314/tjpr.v19i8.13

\title{
Effects of different extracts of Cremastra appendiculata ( $D$. Don) Makino Cremastra appendiculata (D. Don) Makino on apoptosis of A549 cells
}

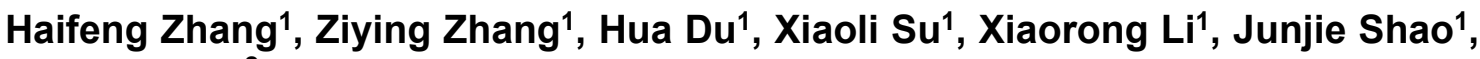 \\ Pengfei Qiao ${ }^{2 *}$ \\ ${ }^{1}$ College of Basic, Inner Mongolia Medical University, Huhehot 010110, ${ }^{2}$ Department of Diagnostic Imaging, Affiliated Hospital \\ of Inner Mongolia Medical University, Huhehot, 010110, PR China \\ *For correspondence: Email: haifengzhang120@126.com
}

Sent for review: 25 March 2020

Revised accepted: 22 July 2020

\begin{abstract}
Purpose: To investigate the effect of different extracts of Cremastra appendiculata (D. Don) Makino on apoptosis of A549 cells, and the underlying mechanism.

Methods: The contents of colchicine in ethyl acetate and n-butanol extracts of Cremastra appendiculata (D. Don) Makino were determined using high performance liquid chromatography (HPLC). Lung cancer A549 cells cultured in vitro were divided into blank control, standard colchicine and Cremastra appendiculata (D. Don) Makino extract groups. The effect of different extract concentrations on proliferation of the cells was determined using methyl thiazolyl diphenyl-tetrazolium (MTT) assay, while apoptosis of $A 549$ cells induced by the extracts was evaluated by flow cytometry (FC).

Results: Compared with the standard colchicine group, there was no colchicine in the n-butanol and ethyl acetate extracts of Cremastra appendiculata. Results from MTT assay showed that the extract inhibited the proliferation of A549 cells $(p<0.05)$. Flow cytometry results showed that ethyl acetate extract significantly enhanced apoptosis in A549 cells $(p<0.05)$. However, $n$-butanol extract had no significant effect on the apoptosis of A549 cells $(p<0.05)$.

Conclusion: The ethyl acetate extract of Cremastra appendiculata (D. Don) Makino induces apoptosis in lung cancer A549 cells. Therefore, there is a need for further research and development of antitumor drugs from the extract of Cremastra appendiculata (D. Don) Makino.
\end{abstract}

Keywords: Cremastra appendiculata (D. Don) Makino, Colchicine, A549 cells, Apoptosis

\begin{abstract}
This is an Open Access article that uses a fund-ing model which does not charge readers or their institutions for access and distributed under the terms of the Creative Commons Attribution License (http://creativecommons.org/licenses/by/4.0) and the Budapest Open Access Initiative (http://www.budapestopenaccessinitiative.org/read), which permit unrestricted use, distribution, and
\end{abstract} reproduction in any medium, provided the original work is properly credited.

Tropical Journal of Pharmaceutical Research is indexed by Science Citation Index (SciSearch), Scopus, International Pharmaceutical Abstract, Chemical Abstracts, Embase, Index Copernicus, EBSCO, African Index Medicus, JournalSeek, Journal Citation Reports/Science Edition, Directory of Open Access Journals (DOAJ), African Journal Online, Bioline International, Open-J-Gate and Pharmacy Abstracts

\section{INTRODUCTION}

The morbidity and mortality of lung cancer are the highest amongst all the malignant tumors, and its incidence is still on the rise globally, especially with respect to lung adenocarcinoma [1-3]. The 5-year survival of patients with lung cancer is only about $16.8 \%$ [4]. Since most patients with lung cancer are not diagnosed until at the advanced stage of cancer, they are treated only with radiotherapy and chemotherapy. At present, the median survival time of patients treated with radiotherapy and chemotherapy is only $8-12$ months, and the adverse effects of 
chemotherapy are obvious. Therefore, it is particularly important to develop highly-efficient and low-toxicity drugs for enhancing the effect of radiotherapy and chemotherapy on lung adenocarcinoma.

Cremastra appendiculata (D. Don) Makino, a popularly used clinical drug in Chinese and Mongolian Tibetan medicine, is frequently used in anticancer therapy [6]. It was first published in Materia Medica [7]. According to the 2010 edition of Chinese Pharmacopoeia, Cremastra appendiculata (D. Don) Makino comes from the orchid plant Azalea. Five new phenanthrene compounds were isolated from the alcohol extract of Azalea by Liu [8]. These compounds exerted cytotoxic effects on colon cancer HCT116, cervical cancer Hela, and breast cancer MDA-MB-231 cells.

Zhou Zhongying, an expert in traditional Chinese medicine, used 453 kinds of drugs in 1374 cancer cases, and 48 types of drugs were used more than 170 times in clinics. The drug with highest frequency of clinical application was Cremastra appendiculata (D. Don) Makino which was used 1062 times, accounting for $77.29 \%$ [6]. Cremastra appendiculata (D. Don) Makino is the most commonly used component in the anticancer prescriptions Yiqi Tudu decoction and mongolian medicine Wumen-17 flavor powder $[9,10]$.

A study by Xia Wenbin reported that compounds isolated from the ethanol extract of Cremastra appendiculata (D.Don) Makino exerted cytotoxicity against human lung cancer A549 cells and breast cancer MCF-7 cells. Results from methyl thiazolyl diphenyl-tetrazolium (MTT) showed that the $\mathrm{IC}_{50}$ values were 17.79 and $12.45 \mathrm{umol} / \mathrm{L}$, respectively [11]. It has been reported that Cremastra appendiculata (D. Don) Makino contains many kinds of alkaloids, including colchicine [12]. Colchicine inhibits mitosis and proliferation of cancer cells by increasing the level of cAMP in these cells. Therefore, it is necessary to study the content of colchicine in Cremastra appendiculata (D. Don) Makino.

It has been reported that Cremastra appendiculata (D. Don) Makino extract induces apoptosis of tumor cells by upregulating the contents of Cyt-C, Bax and Caspase-3, and downregulating the protein expression of $\mathrm{Bcl}-2$ [13]. The purpose of this study was to determine the effect of different extracts of Cremastra appendiculata (D. Don) Makino on apoptosis of human lung cancer A549 cells.

\section{EXPERIMENTAL}

\section{Drugs and reagents}

Human lung cancer A549 cells were obtained from Shanghai Institute of Cell Biology, Chinese Academy of Sciences. Cremastra appendiculata (D. Don) Makino was purchased from Hebei Province Yaoxing Medicinal Materials Co. Ltd. Colchicine Standard was bought from China Institute of Pharmaceutical and Biological Products Control, while MTT Reagent was product of Sigma Company. Fetal Bovine Serum was purchased from Gibco Company. TrypsinEDTA Digestive fluid was bought from Hyclone Company. Cell Culture bottles and 96-well Culture Plate were products of Corning Company. High performance liquid Chromatography was purchased from HewlettPackard. Annexin V FITC/PI detection kit and flow cytometry were products of BD Company, while $\mathrm{CO}_{2}$ incubator was purchased from American Thermo Company.

\section{Experimental groups}

The cells were divided into blank control group, and groups treated with different concentrations of ethyl acetate (Et-Ac groups), different concentrations of n-butanol extracts group (n-Bu groups), and as well as a group treated with colchicine standard (control group).

\section{Drug extraction}

The crude drug of Cremastra appendiculata (D. Don) Makino (1 kg) was refluxed and extracted thrice with $75 \%$ ethanol (2 hours each). The extract of Cremastra appendiculata (D. Don) Makino was obtained by combining the extracts, concentrating under reduced pressure, and extracting with ethyl acetate and n-butyl alcohol in turn, and drying. The extract was dissolved in DMSO, mixed with mother liquor, and sterilized by filtration through a $0.22-\mu \mathrm{m}$ filter. Prior to experimental application, the culture medium was diluted to the desired concentration.

\section{High performance liquid chromatography}

The n-butanol extract $(0.1 \mathrm{~g})$ was mixed with $25 \mathrm{~mL}$ of methanol, and subjected to ultrasonication for $630 \mathrm{~min}$, followed by filtration through a microporous membrane. Then, $20 \mu \mathrm{L}$ mixed solution was injected into the sample. The ethyl acetate extract was similarly treated. The HPLC conditions were: flow rate of $1.0 \mathrm{~mL} / \mathrm{min}$, detection wavelength of $220 \mathrm{~nm}$, column temperature of $40{ }^{\circ} \mathrm{C}$, 
injection volume of $10 \mu \mathrm{L}$, and concentration of reference standard set as $59 \mu \mathrm{g} / \mathrm{mL}$ (methanol).

\section{MTT assay}

The A549 cells were cultured in RPMI containing $10 \%$ fetal bovine serum in an incubator with $5 \%$ $\mathrm{CO}_{2}$ at $37{ }^{\circ} \mathrm{C}$. When the cells grew to $90 \%$ of the bottom area of the culture medium, they were digested with $0.05 \%$ trypsin and passaged at $1: 3$.

Cells at logarithmic growth phase were selected, adjusted to a density of $4 \times 10^{4}$ cells $/ \mathrm{mL}$, and inoculated and incubated in 96-well culture plate (100 $\mu \mathrm{L} /$ well) for 24 hours. Different concentrations of Cremastra appendiculata (D. Don) Makino extract were added and cultured for 20 hours, followed by MTT colorimetric analysis. The MTT solution $(5 \mathrm{~g} / \mathrm{L})$ was added to the well plate and cultured at $37{ }^{\circ} \mathrm{C}$ for 4 hours. Thereafter, the culture medium was replaced with DMSO $(150 \mu \mathrm{L} /$ well), and the wells were shaken for $10 \mathrm{~min}$ to dissolve the formazan crystals formed. Thereafter, the absorbance of each well was read at $490 \mathrm{~nm}$, and the inhibition was calculated as in Eq 1.

$H(\%)=\{(\mathrm{Ac} \mathrm{At}) / \mathrm{Ac}\} 100$

where Ac and At are the absorbance of control and test/experimental groups, respectively.

\section{Flow cytometry}

Cells at logarithmic at growth phase were digested with $0.25 \%$ trypsin and collected, and then moved into the incubator for $24 \mathrm{~h}$ to make the cells adhere to the wall. The cells were cultured with blank control group, high-dose group, middle-dose group and low-dose group for $24 \mathrm{~h}$. When the cell density reached $1 \times 10^{6}$ cells/mL/well, the cell suspensions were washed twice with PBS. The \% apoptosis of cells was calculated according to the manufacturer's operating instructions as indicated in Annexin V/PI double staining kit. In essence, $500 \mu \mathrm{L}$ FITC-labeled Annexin $\mathrm{V}$ solution and $5 \mu \mathrm{L}$ of propidium iodide $(\mathrm{PI})$ solution were added and incubated in the dark for $15 \mathrm{~min}$. Then, \% apoptosis was quantitatively analyzed using flow cytometry.

\section{Statistical analysis}

Statistical analysis was done with SPSS 19.0. Data are expressed as mean \pm standard deviation (SD). Two-group comparison was carried out using t-test. Values of $p>0.05$ were considered statistically significant.

\section{RESULTS}

\section{Colchicine content}

Ethyl acetate extract and n-butanol extract were obtained after evaporating the solvents under reduced pressure. The chromatographic results of colchicine content in the extracts determined using HPLC are shown in Figure 1.
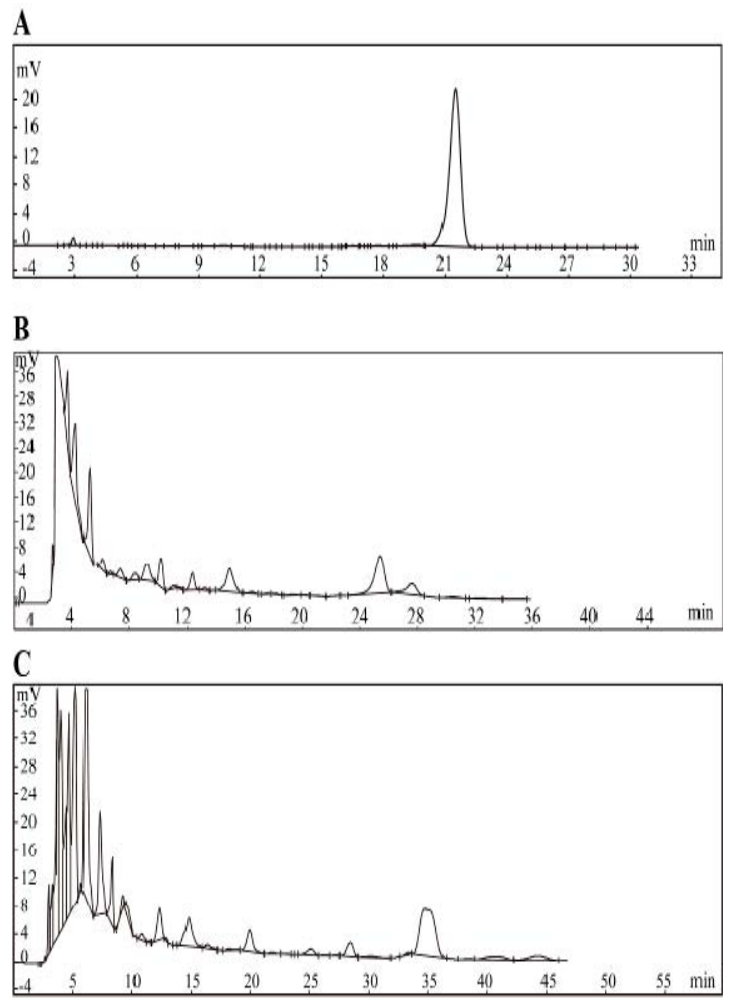

Figure 1: HPLC peaks of EtAc and n-butanol extracts of Cremastra appendiculata (D. Don) Makino. Absorption spectra showing colchicine content of control sample (A); colchicine content of n-butanol extract $(B$, and colchicine content of ethyl acetate extract (C)

\section{Inhibitory effect of Cremastra appendiculata (D. Don) Makino extracts on A549 cells}

The ethyl acetate and n-butanol extracts of Cremastra appendiculata (D. Don) Makino had different inhibitory effects on A549 cells. The percentage inhibition by Et Ac $(1250 \mathrm{ug} / \mathrm{ml})$ on A549 cells was $92.4 \%$, while the effects of different concentration gradients of n-Bu on A549 cells were similar. These results are shown in Figure 2. 


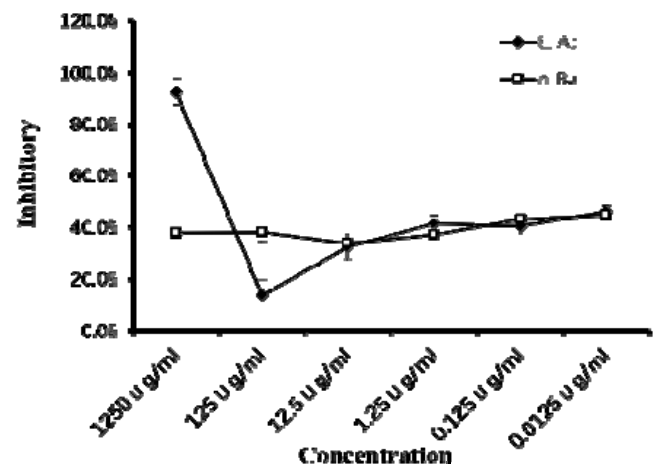

Figure 2: Inhibitory effects of ethyl acetate and nbutanol extracts on A549cells, as determined using MTT assay. Et Ac = ethyl acetate extract of Cremastra appendiculata (D. Don) Makino in blank control group; $\mathrm{n}-\mathrm{Bu}=\mathrm{n}$-butanol extract of Cremastra appendiculata (D. Don) Makino

\section{Effect of Cremastra appendiculata (D. Don) Makino extract on apoptosis of A549 cells}

The results in Figure 3 and Figure 4 show that different concentrations of $\mathrm{E}_{\mathrm{t}} \mathrm{A}_{\mathrm{c}}$ extract significantly increased percentage early apoptosis and total apoptosis of A549 cells $(p<$ 0.05 ), but the effects of different concentrations of $\mathrm{n}-\mathrm{Bu}$ extract on early apoptosis and total apoptosis of A549 cells were not significantly different from those of the blank control group $(p$ $>0.05$ ).

\section{DISCUSSION}

Although it has been reported in the literature that Cremastra appendiculata (D. Don) Makino is rich in colchicine, this alkaloid was not detected by high performance liquid chromatography (HPLC) in the present study. The reason for this inconsistency with literature reports may be related to the differences in the origin of Cremastra appendiculata (D. Don) Makino used. Lijiang Mountain Cremastra appendiculata (D. Don) Makino was used in the literature reports, while Hebei Cremastra appendiculata (D. Don) Makino was used in the present study. The ethanol extract of Cremastra appendiculata (D. Don) Makino can be re-extracted with petroleum ether, ethyl acetate and n-butanol. Although the anti-LA795 cell effects of some petroleum ether monomers were screened using MTT test in vitro, the doses used were large. Due to the fact that the total amount of petroleum ether extract was small, it was difficult prepare solutions using the petroleum ether extract. Therefore, only $E_{t} A_{c}$ and $n-B u$ extract were chosen for use in this research.
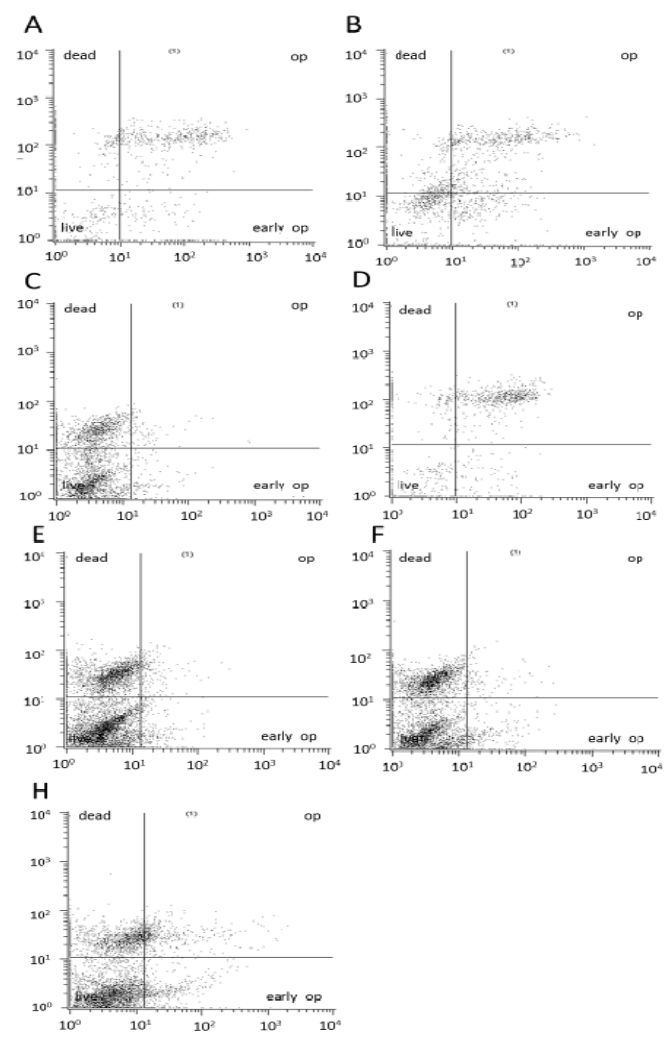

Figure 3: Effect of the different extracts on A549 cell apoptosis, as determined using flow cytometry. A: control; B: $E_{t} A_{c} 125 \mu \mathrm{g} / \mathrm{mL}$; $C: E_{t} A_{c} 12.5 \mu \mathrm{g} / \mathrm{mL}$; D: Et Ac $1.25 \mu \mathrm{g} / \mathrm{mL}$; E: n-Bu $1250 \mu \mathrm{g} / \mathrm{mL}$; F: n-Bu 125 $\mu \mathrm{g} / \mathrm{mL} ; \mathrm{H}: \mathrm{n}-\mathrm{Bu} 12.5 \mu \mathrm{g} / \mathrm{mL}$

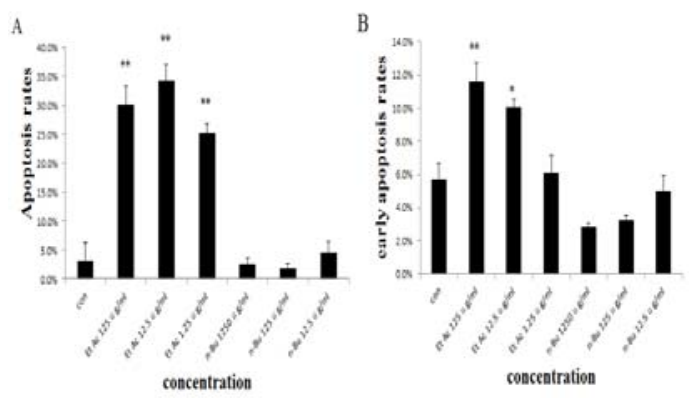

Figure 4: Effect of different extracts on apoptosis of A549 cell, as determined using flow cytometry. A: Effect of Et Ac and n-Bu extracts on apoptosis of A549 cells; B: effect of ethyl acetate and n-butanol extracts of Cremastra appendiculata (D. Don) Makino on early apoptosis of A549 cells. ${ }^{*} p<0.01,{ }^{*} p<0.05$, compared with the blank control group

The results showed that the extracts of Cremastra appendiculata (D. Don) Makino had different inhibitory effects on A549 cells. The percentage inhibition of $E_{t} A_{c}$ extract (1250 $\mathrm{ug} / \mathrm{ml}$ ) on A549 cells was $92.4 \%$. Hence, the concentration of Et Ac extract chosen was less than $1250 \mu \mathrm{g} / \mathrm{mL}$. The percentage inhibition 
values of different concentrations of $\mathrm{n}-\mathrm{Bu}$ on A549 cells were similar, so a high dose was started with. It has been reported in the literature that Xiaoai jiedu recipe used in clinics inhibited the growth of lung cancer cells in nude mice by reducing the content of serum VEGF [14]. Research on Cremastra appendiculata (D. Don) Makino is mainly focused on its water decoctions and polysaccharides. The inhibitory effect of Cremastra appendiculata (D. Don) Makino on breast cancer and lung cancer cells in mice have been demonstrated via MTT assay $[15,16]$.

Cremastra appendiculata (D. Don) Makino polysaccharides exerted anti-tumor effects by activating NK cells and inducing innate immune response of breast and lung cancer cells [17]. Phenanthrene and dihydrophenanthrene, and bibenzyl compounds contained in the ethanol extract of Cremastra appendiculata (D. Don) Makino exert cytotoxicity and anti-angiogenesis [18]. These may also be important chemical bases for its anticancer effect. In this study, in order to determine the anti-tumor effect of Cremastra appendiculata (D. Don) Makino, its ethanol extract was further fractionated into $E_{t} A_{c}$ and $n-$ Bu extracts.

Bibenzyl compounds are present in the $E_{t} A_{c}$ extract of Cremastra appendiculata (D. Don) Makino [19]. These compounds exerted significant cytotoxicity against small cell lung cancer $\mathrm{NCl}-\mathrm{H} 187$ cells [20]. It has been reported that the $E_{t} A_{c}$ extract exerted inhibitory effect on LA795 cell line (mouse lung adenocarcinoma cell line, as confirmed using MTT assay). Cymbidium is one of the sources of Cremastra appendiculata (D. Don) Makino. Gastrodia elata aglycone in n-butanol extract of Cremastra appendiculata (D. Don) Makino produced a good anti-tumor effect by inhibiting the production of nitric oxide (NO) and inducible nitric oxide synthase (iNOS) expression in lipopolysaccharide-activated RAW264.7 macrophages [21]. This is also an important reason why researchers choose the n-butanol extract of Cremastra appendiculata (D. Don) Makino. However, literature reports suggest that the n-BuAc extract produced no anti-LA795 cell effects at both high and low concentrations, which is slightly different from the results of the present study [21].

At present, there are no related literature reports in China and abroad on the direct anti-lung cancer effect of Cremastra appendiculata (D. Don) Makino extract with respect to apoptosis of lung cancer cells. Apoptosis is a process of cell death controlled by genes in organisms. It leads to uncontrolled growth, excessive proliferation and tumorigenesis. Therefore, enhancement of apoptosis of A549 cells is helpful in inhibiting the occurrence and progression of tumor. Giard et al first established the A549 cell model [22]. This model was derived from lung adenocarcinoma of human alveolar cells, making A549 cells the most widely used in the study of lung cancer [23].

This study found that Cremastra appendiculata (D. Don) Makino promoted the apoptosis of lung cancer A549 cells, thus delaying the progression of lung cancer. In order to understand the mechanism involved in the anticancer effects of $E_{t} A_{c}$ and $n-B u$ extracts against $A 549$ cells, the effects of different concentrations of $E_{t} A_{c}$ and $n$ $\mathrm{Bu}$ extracts on A549 cells were determined. It was found that the $E_{t} A_{c}$ extract significantly inhibited increase in early apoptosis and total apoptosis of A549 cells, while n-Bu extract had no obvious effect on A549 cells.

These results showed that the inhibitory effect of $E_{t} A_{c}$ extract on the growth of A549 cells was consistent with that reported by Xia Wenbin through MTT assay. However, the latter did not mention the effect of $n-B u$ extract on A549 cell apoptosis. This study was aimed at investigating the anti-lung cancer effect of Cremastra appendiculata (D. Don) Makino, and the underlying mechanism. This would also unravel its anti-lung adenocarcinoma effect, thereby providing a pharmacological basis for the clinical development and utilization of Cremastra appendiculata (D. Don) Makino, and pharmacological basis for the development of ethnic medicine.

\section{CONCLUSION}

The findings of this study show that the ethyl acetate extract of Cremastra appendiculata (D. Don) Makino $E_{t} A_{c}$ promotes apoptosis of lung cancer A549 cells. This finding provides a basis for further investigation of antitumor drugs related to Cremastra appendiculata (D. Don) Makino.

\section{DECLARATIONS}

\section{Acknowledgement}

This work was supported by Scientific Research Program of Colleges and Universities in Inner Mongolia Autonomous region (NJZZ18100), and Science and Technology million Project of Inner Mongolia Medical University (nos. YKD2016KJBW018 and YKD2017KJBW007). 


\section{Conflict of interest}

No conflict of interest is associated with this work.

\section{Contribution of authors}

We declare that this work was done by the authors named in this article, and all liabilities pertaining to claims relating to the content of this article will be borne by the authors. All authors read and approved the manuscript for publication. Ziying Zhang and Haifeng Zhang conceived and designed the study. Hua Du, xiaoli Su, Junjie Shao, Pengfei Qiao and Xiaorong $\mathrm{Li}$ collected and analyzed the data, while Ziying Zhang wrote the manuscript. Ziying Zhang and Haifeng Zhang contributed equally.

\section{Open Access}

This is an Open Access article that uses a funding model which does not charge readers or their institutions for access and distributed under the terms of the Creative Commons Attribution License (http://creativecommons.org/licenses/by/ 4.0) and the Budapest Open Access Initiative (http://www.budapestopenaccessinitiative.org/rea d), which permit unrestricted use, distribution, and reproduction in any medium, provided the original work is properly credited.

\section{REFERENCES}

1. Ferlay J, Shin HR, Bray F, David F, Colin M, Donald MP. Estimates of worldwide burden of cancer in 2008: GLOBOCAN 2008. Int J Cancer 2010; 127(12): 28932917.

2. Chen $W$, Zheng $R$, Zhang $S$, Ping $Z$, Hongmei $Z$, Xiaonong $Z$, Jie $H$. Annual report on status of cancer in China 2010. Chin J Cancer Res 2014; 6(1): 48-58.

3. Denisenko TV, Budkevich IN, Zhivotovsky B. Cell deathbased treatment of lung adenocarcinoma. Cell Death Dis 2018; 9(2): 117.

4. Wender R, Fontham $E T$, Barrera $E J r$, Stacey AF, Deana MB, Debbie S, Otis WB, Richard CW. American Cancer Society lung cancer screening guidelines. CA Cancer J Clin 2013; 63(2): 107-117.

5. Shackelford RE, Vora M, Mayhall K, James C. ALKrearrangements and testing methods in non-small cell lung cancer: a review. Genes Cancer 2014; 5(2): 1-14

6. Zhao Hui, Wang Zhiying, Zhou Zhongying. Analysis of Herb Used in Malignant Tumor Treatment Based on Phlegm Syndrome Differentiation by ZHOU Zhongying. J Tradit Chin Med 2015, 56(9): 740-742.

7. Qi Li, Yuzong Chen, Hailiang Xin, Peiken Xiao. Advances in studies on chemical constituents in plants of Pseudobulbus Cremastrae seu Pleiones and their pharmacological activities. Chin Tradit Herb Drugs 2007; (11): 1734-1738.

8. Liu L, Li J, Zeng $K W$, Yong J, Tu PF. Five New Biphenanthrenes from Cremastra appendiculata. Molecules 2016; 21(8): 1089-1098.

9. Wu Chao, Ma Dongye. Clinical observation on the treatment of 234 cases of advanced esophageal and Cardiac carcinoma with Mongolian Medicine Wumen-17 Weisan. J Med Pharm Chin Minor 2001; 7(2): 12-13.

10. Yi Ji, Mianhua Wu. Research Progress of Pseudobulbus Cremastrae seu Pleiones on Chemical Composition and Antitumor Mechanisms. Chin Arch of Tradit Chin Med 2018; 36(3): 596-598.

11. Xia WB, Xue Z, Li S, Wang SJ, Yang YC, He DX, Ran GL, Kong LZ, Shi JG. Study on the chemical composition and cytotoxic activity of azalea. Chin J Chin Mater Medic 2005; 30(23): 1827-1830.

12. Shi Yao, Yang Yaling, Liu Mousheng. Extraction the Colchicine of Iphigenia indica in Aqueous Two-phase System. Nat Prod Res Dev 2012; 24: 1412-1416.

13. Wisbeck E, Facchini JM, Alves EP, Marcia LL, Regina $M M$, Jorge $L N$, Sandra AF. A polysaccharide fraction extracted from Pleurotus ostreatus mycelial biomass inhibit Sarcoma 180 tumor. An Acad Bras Cienc 2017; 89(3 Suppl): 2013-2020.

14. Jiping Zhu, Jian Shen, Tingting Yan. Inhibitory Effect of Xiao'ai Jiedu Recipe on Lung Cancer A549Cell Induced Xenograft Nude Mice Model. J Sichuan Tradit Chin Med 2015; 33 (5): 41-44.

15. Xiaojuan Xu, Zhihan Zhou, Yu Mao, Fang Xu, Jie Li, Jianhua He. Study the Effect of Pleione yunnanensis Rolfe Polysaccharides on $I L-2$ and 053 Protein Expression. Food Res Dev 2016; 18: 6-10.

16. Xiaoyu Niu, Lu Wang, Fang Sun, Yongjie Gong, Chun Guo, Xiuhua Hu. Effect of Pleurotus ostreatus decoction on breast cancer MDA-MB-231 cells. Chin Tradit Pat Med 2018; 40(1): 197-200

17. El-Deeb NM, El-Adawi HI, El-Wahab AEA, Ahmed MH, Hesham A El, Enshasy, YW, Keith RD. Modulation of NKG2D, KIR2DL and Cytokine Production by Pleurotus ostreatus Glucan Enhances Natural Killer Cell Cytotoxicity Toward Cancer Cells. Front Cell Dev Biol 2019; 7: 165.

18. Yi Dai, Longru Sun. Advances in studies on bibenzyls naturally occurred in plant. Chin Tradit Herb Drugs 2008; 39(11): 1753-1757.

19. Liu L, Yin QM, Yan X, Chen H, Wang W, Wang RK, Luo $X$, Zhang XW. Bioactivity-Guided Isolation of Cytotoxic Phenanthrenes from Spiranthes sinensis. J Agric Food Chem 2019; 67(26): 7274-7280.

20. Apisantiyakom S, Kittakoop $P$, Manyum T, Kirtikara K, John BB, Thebtaranonth Y. Novel Biologically Active Bibenzyls From Bauhinia Saccocalyx Pierre. Chem Biodivers 2004; 1(11): 1694-1701.

21. Liu XQ, Li XP, Yuan WK, Yuan QY, Qin BH. Two New Phenanthrene Glucosides from Cremastra appendiculata and their Cytotoxic Activities. Nat Prod Commun 2016; 11(4): 477-479.

Trop J Pharm Res, August 2020; 19(8): 1658 
22. Giard DJ, Aaronson SA, Todaro G J, P Arnstein, JH Kersey, H Dosik, WP Parks. In vitro cultivation of human tumors: establishment of cell lines derived from a series of solid tumors. J Natl Cancer Inst 1973; 51(5): 1417.

23. Cooper JR, Abdullatif MB, Burnett EC, Karen E Kempsell, Franco Conforti, Howard Tolley, Jane E Collins, Donna
E Davies. Long Term Culture of the A549 Cancer Cell Line Promotes Multilamellar Body Formation and Differentiation towards an Alveolar Type II Pneumocyte Phenotype. Plos One 2016; 11(10): e0164438. 